\title{
Integrated Prefetching and Caching in Single and Parallel Disk Systems
}

\author{
Susanne Albers \\ Institute for Computer Science \\ Freiburg University \\ Georges-Köhler-Allee 79 \\ 79110 Freiburg, Germany \\ salbers@informatik.uni-freiburg.de
}

\author{
Markus Büttner \\ Institute for Computer Science \\ Freiburg University \\ Georges-Köhler-Allee 79 \\ 79110 Freiburg, Germany \\ buettner@informatik.uni-freiburg.de
}

\begin{abstract}
We study integrated prefetching and caching in single and parallel disk systems. There exist two very popular approximation algorithms called Aggressive and Conservative for minimizing the total elapsed time in the single disk problem. For $D$ parallel disks, approximation algorithms are known for both the elapsed time and stall time performance measures. In particular, there exists a $D$-approximation algorithm for the stall time measure that uses $D-1$ additional memory locations in cache.

In the first part of the paper we investigate approximation algorithms for the single disk problem. We give a refined analysis of the Aggressive algorithm, showing that the original analysis was too pessimistic. We prove that our new bound is tight. Additionally we present a new family of prefetching and caching strategies and give algorithms that perform better than Aggressive and Conservative.

In the second part of the paper we investigate the problem of minimizing stall time in parallel disk systems. We present a polynomial time algorithm for computing a prefetching/ caching schedule whose stall time is bounded by that of an optimal solution. The schedule uses at most $3(D-1)$ extra memory locations in cache. This is the first polynomial time algorithm for computing schedules with a minimum stall time. Our algorithm is based on the linear programming approach of [1]. However, in order to achieve minimum stall times, we introduce the new concept of synchronized schedules in which fetches on the $D$ disks are performed completely in parallel.
\end{abstract}

\section{Categories and Subject Descriptors}

F.2 [Analysis of Algorithms and Problem Complex-

*Work supported by the Deutsche Forschungsgemeinschaft, project project AL $464 / 3-1$, and by the EU, projects APPOL and APPOL II.

Permission to make digital or hard copies of all or part of this work for personal or classroom use is granted without fee provided that copies are not made or distributed for profit or commercial advantage and that copies bear this notice and the full citation on the first page. To copy otherwise, to republish, to post on servers or to redistribute to lists, requires prior specific permission and/or a fee.

SPAA'03, June 7-9, 2003, San Diego, California, USA.

Copyright 2003 ACM 1-58113-661-7/03/0006 ...\$5.00. ity]: Nonnumerical algorithms and problems

\section{General Terms}

Algorithms

\section{Keywords}

Magnetic disk systems, prefetching, caching

\section{INTRODUCTION}

In today's computer systems there is a growing gap between processor speed and memory access time. Therefore an effective utilization of caches is increasingly important. Prefetching and caching are well-known and extensively studied techniques to improve the performance of memory hierarchies. In prefetching missing memory blocks are loaded from slow memory, e.g. a disk, into cache before their actual reference. Caching strategies try to keep actively referenced blocks in cache. The goal of both tools is to reduce processor stall times that are incurred when requested data is not available in cache. Most of the previous work on prefetching and caching investigated these two techniques separately, see e.g. [3, 4, 7, 8, 15, 18, 19] for some selected papers, although there is a strong correlation. Prefetching blocks too early can cause the eviction of blocks from cache referenced in the near future. Caching blocks too long can diminish the effect of prefetching.

In recent years, initiated by a paper of Cao et al. [5], there have been a number of studies that integrate prefetching and caching. The goal is to design strategies that coordinate prefetching and caching decisions. Both theoretical and experimental studies were presented $[5,6,9,10,11,12$, $13,14,16,17]$. It was demonstrated that an integration of prefetching and caching leads to a substantial improvement in systems performance.

Cao et al. [5] introduced a model for integrated prefetching and caching that we will also use in this paper. We are given a request sequence $\sigma=r_{1}, \ldots, r_{n}$ consisting of $n$ requests. Each request specifies a block in the memory system. We first assume that all blocks reside on a single disk. To serve a request the requested block must be in cache. The cache can simultaneously store $k$ blocks. Serving a request to a block in cache takes 1 time unit. If a requested block is not in cache, then it must be fetched from disk, which takes $F$ time units. A fetch operation may overlap with the service of 
requests to blocks already in cache. If a fetch, i.e. a prefetch, of a block is initiated at least $F$ requests before the reference to the block, then the block is in cache at the time of the request and no processor stall time is incurred. If the fetch is started only $i, i<F$, requests before the reference, then the processor has to stall for $F-i$ time units until the fetch is finished. When a fetch operation is initiated, a block must be evicted from cache to make room for the incoming block. Thus a prefetch operation critically affects the cache configuration in that we must also drop a block. The goal is to minimize the total processor stall time incurred on the entire request sequence. This is equivalent to minimizing the elapsed time, which is the sum of the processor stall time and the length of the request sequence. We point out here that the input $\sigma$ is completely known in advance.

To illustrate the problem, consider a small example. Let $\sigma=b_{1}, b_{2}, b_{3}, b_{4}, b_{4}, b_{5}, b_{1}, b_{4}, b_{4}, b_{2}$. Assume that we have a cache of size $k=4$ and that initially blocks $b_{1}, b_{2}, b_{3}$ and $b_{4}$ reside in cache. Let $F=4$. The first missing block is $b_{5}$. We could initiate the fetch for $b_{5}$ when starting the service of the request to $b_{2}$. The fetch would be executed while serving requests $b_{2}, b_{3}, b_{4}$ and $b_{4}$ and completed in time. However, when starting this fetch, we can only evict $b_{1}$, which is requested again after $b_{5}$. To load $b_{1}$ we incur 3 units of stall time as the fetch can only overlap with the request to $b_{5}$. A better option is to start the fetch for $b_{5}$ at the request to $b_{3}$. We generate 1 unit of processor stall before the request to $b_{5}$ but can evict $b_{2}$, which is requested again only at the end of $\sigma$ and can be fetched back without incurring any stall time. The stall time of this second solution is 1 time unit and the elapsed time is 11 time units.

Integrated prefetching and caching is equally interesting in parallel disk systems. Suppose that we have $D$ disks and that each memory block resides on exactly one of the disks. Blocks from different disks may be fetched in parallel. When starting a fetch, we can evict any block from cache, which corresponds to the case that blocks are read-only and do not have to be written back to disk. Of course we can take advantage of the parallelism given by a multiple disk system. If the processor incurs stall time to wait for the completion of a fetch, then other fetches executed in parallel also make progress towards completion during that time. Again we wish to minimize the total stall time or elapsed time.

As an example consider two disks, where $b_{1}, b_{2}, b_{3}$ and $b_{4}$ reside on disk 1 and $c_{1}, c_{2}$ and $c_{3}$ reside on disk 2. Again $k=4$ and $F=4$. Suppose that initially $b_{1}, b_{2}, c_{1}$ and $c_{2}$ are in cache and that $\sigma=b_{1}, b_{2}, c_{1}, c_{2}, b_{3}, c_{3}, b_{4}$. Disk 1 initiates a fetch for $b_{3}$ at the request to $b_{2}$; it evicts $b_{1}$. Disk 2 starts a fetch for $c_{3}$ one request later and evicts $b_{2}$. Disk 1 starts a second fetch at the request to $b_{3}$ in order to load $b_{4}$. There is 1 unit of stall time before the request to $b_{3}$. The fetch on disk 2 benefits from this time unit so that no additional stall time is generated before the request to $c_{3}$. The second fetch on disk 1 incurs 2 units of stall time. The total stall time of this solution is equal to 3 time units.

Previous work: Cao et al. [5] introduced two popular algorithms, called Conservative and Aggressive, for integrated prefetching and caching in the single disk problem. Conservative performs exactly the same block replacements as the optimum offline paging algorithm MIN [3], while initiating a fetch at the earliest point in time that is consistent with the choice of blocks to be evicted. Cao et al. showed that Conservative achieves an approximation ratio of 2 with respect to the elapsed time performance measure, i.e. the elapsed time of Conservative's schedule is at most twice the elapsed time of an optimal schedule. This bound is tight. The $A g$ gressive algorithm starts prefetch operations as soon as possible. Whenever the algorithm is not in the middle of a fetch, it initiates a new fetch provided it can evict a block from cache that is not requested before the block to be fetched. Cao et al. proved that the approximation ratio, with respect to elapsed time, is at most $\min \{1+F / k, 2\}$ and that this ratio is tight for $F \geq k$. Kimbrel and Karlin [12] analyzed Aggressive and Conservative in parallel disk systems and showed that the approximation ratios are essentially equal to $D$. They also proposed an algorithm Reverse Aggressive, which is the Aggressive algorithm on the reverse sequence, and proved that the approximation guarantee is bounded by $1+D F / k$. Again the approximation ratios are with respect to the elapsed time measure. Extensive experimental studies, in particular on the performance of the Aggressive algorithm, were presented in $[5,6,13,14]$.

It was shown in [1] that optimal prefetching/caching schedules for a single disk can be computed in polynomial time. The idea is to formulate the prefetching and caching problem as a linear program and to prove that there exists an optimal solution that is integral. The approach was extended to parallel disk systems and gave a $D$-approximation algorithm for the stall time performance measure if the algorithm may use $D-1$ extra memory locations in cache. Note that approximating stall time is harder than approximating elapsed time because in the stall time measure the length of the request sequence is not part of the objective function. In [2] it was shown that the linear program of [1] can be translated directly into a multicommodity flow problem.

Our contribution: We investigate approximation algorithms for single disk systems as well as algorithms for computing optimal schedules in parallel disk systems. In Section 2 we study the single disk problem and first present a refined analysis of the Aggressive algorithm, showing that the analysis by Cao et al. [5] was too pessimistic. We prove that Aggressive achieves an approximation ratio of $\min \{1+$ $\left.F /\left(k+\left\lfloor\frac{k}{F}\right\rfloor-1\right), 2\right\}$ in the elapsed time measure. Compared to the bound of Cao et al. there is an additional $\left\lfloor\frac{k}{F}\right\rfloor-1$ in the denominator of the first term. If $k / F$ is large, which is true in most practical applications, the new bound is much lower. Kimbrel and Karlin [12] mentioned that in practice $k / F$ is typically at least 200 . We also show that our analysis is tight. For any $F$ and $k$, the approximation ratio of Aggressive is in general not smaller than $\min \left\{1+F /\left(k+\frac{k-1}{F-1}\right), 2\right\}$. Since Aggressive is the most popular algorithm for integrated prefetching and caching, it is important to know its true approximation guarantee.

We also give improved approximation ratios if $k / F$ is small. More generally, we present a new family of algorithms for integrated prefetching and caching. The algorithms, called Delay(d), delay the next fetch operation for $d$ time units, for any fixed non-negative integer $d$. Setting $d=0, \operatorname{Delay}(d)$ is equal to the standard Aggressive strategy; for $d=|\sigma|$ we obtain Conservative. Hence our family of algorithms bridges the gap between the two classical algorithms for prefetching and caching. As mentioned above, fetching blocks too early can have a negative influence on the cache configuration and reduce the effective size of the cache. Thus it is natural to investigate the effect of delaying fetches by some time units. We analyze Delay(d) for any 
$d$ and show that, surprisingly, the best choice of $d$ gives an approximation ratio of $\sqrt{3} \approx 1.73$. Combining this strategy with Aggressive, we obtain an algorithm that achieves an approximation ratio of $\min \left\{1+F /\left(k+\left\lfloor\frac{k}{F}\right\rfloor-1\right), \sqrt{3}\right\}$ and hence performs better than both Conservative and Aggressive.

In Section 3 we investigate the problem of minimizing stall time on $D$ parallel disks. We present a polynomial time algorithm that, given a request sequence $\sigma$, computes a schedule whose stall time is bounded by that of an optimal solution for $\sigma$. The solution uses at most $3(D-1)$ extra memory locations in cache. In practice $D$ is small, typically 4 or 5 . Thus at the expense of slightly increasing the extra memory resources, we are able to improve the best previous approximation guarantee from $D$ to 1 . In fact our algorithm is the first polynomial time strategy for computing schedules with a minimum stall time. Our algorithm is based on the linear programming approach of [1]. However, in order to obtain solutions with a smaller stall time, we introduce the new concept of synchronized schedules in which fetches on the $D$ disks are performed completely in parallel. We show that there exist synchronized schedules that achieve a minimum stall time provided that they may use $D-1$ extra memory locations in cache. Using linear programming we then compute an optimal synchronized solution that uses $D-1$ extra cache locations. Applying techniques from [1] we transform an optimal fractional solution into an integral solution. Compared to [1], our transformation algorithm must use a different scheme for assigning blocks to be evicted in the integral solution.

\section{APPROXIMATION ALGORITHMS FOR A SINGLE DISK}

Throughout this section approximation ratios refer to the elapsed time performance measure. The Aggressive algorithm works as follow. Whenever the algorithm is not prefetching a block, it initiates a prefetch for the next missing block in the sequence provided it can evict a block from cache that is not requested before the block to be fetched. In this case it evicts the block whose next reference is furthest in the future.

Theorem 1. The approximation ratio of Aggressive is at most $\min \left\{1+F /\left(k+\left\lfloor\frac{k}{F}\right\rfloor-1\right), 2\right\}$.

Our upper bound proofs in this section are based on the dominance concept introduced by Cao et al. [5]. Given a request sequence $\sigma=r_{1}, \ldots, r_{n}$ consisting of $n$ requests and a prefetching algorithm $A$, let $c_{A}(A)$ be the index of the next request at time $t$ when $A$ serves $\sigma$. Let $H_{A}(i)$ be the set of blocks not present in $A$ 's cache when the next reference is $r_{i}$. Let $h_{A}(i, j), h_{A}(i, j) \geq i$, be the smallest index such that exactly $j$ different blocks in $H_{A}(i)$ are referenced in the subsequence consisting of request $i$ up to (and including) request $h_{A}(i, j)$. Intuitively, $h_{A}(i, j)$ is the index of the first reference to the $j$-th block not present in cache after $r_{i-1}$. Index $h_{A}(i, j)$ is also referred to as $A$ 's $j$-th hole. The parameter $j$ varies between 1 and $n-k$. Given two prefetching algorithms $A$ and $B, A$ 's cursor at time $t$ dominates $B$ 's cursor at time $t^{\prime}$ if $c_{A}(t) \geq c_{B}\left(t^{\prime}\right)$. We say that $A$ 's holes at time $t$ dominate $B$ 's holes at time $t^{\prime}$ if $h_{A}\left(c_{A}(t), j\right) \geq h_{B}\left(c_{B}\left(t^{\prime}\right), j\right)$ for all $j$. Combining these two definitions we say that $A$ 's state at time $t$ dominates $B$ 's state at time $t$ ' if $A$ 's cursor at time $t$ dominated $B$ 's cursor at time $t^{\prime}$ and $A$ 's holes at time $t$ dominate $B$ 's holes at time $t^{\prime}$. Cao et al. [5] proved the following domination lemma.

Lemma 1. Suppose that algorithm A (resp. algorithm B) initiates a prefetch at time $t$ (resp. $t^{\prime}$ ) and both algorithms prefetch the next missing block and replace the block whose next reference is furthest in the future. Suppose that $A$ 's state at time $t$ dominates $B$ 's state at time $t^{\prime}$. Then $A$ 's state at time $t+F$ dominates $B$ 's state at time $t^{\prime}+F$.

Proof of Theorem 1 . We assume $F \leq k$ and prove an upper bound of $1+F /\left(k+\left\lfloor\frac{k}{F}\right\rfloor-1\right)$ on Aggressive's approximation ratio. If $F>k$, then our bound implies a 2approximation, which was already shown by Cao et al. [5]. The global structure of our proof is similar to that of Cao et al. and we describe the difference. Let OPT be an optimal prefetching algorithm. We partition the given request sequence into phases such that each phase consists of exactly $k+\left\lfloor\frac{k}{F}\right\rfloor-1$ consecutive requests. We prove by induction on the number of phases that the following invariant holds. During each phase $i$ there is a time $t$ such that Aggressive's state at time $t$ dominates OPT's state at time $t^{\prime} \geq t-i F$. This implies that Aggressive needs at most $F$. (number of phases) more time units than OPT to serve the entire request sequence, and on the average it spends at most $F$ extra time units in each phase. Cao et al. divided the request sequence into different phases which consisted potentially of only $k$ requests. This resulted in a higher upper bound.

To establish the invariant consider a phase $i$ and assume that at time $t$ during phase $i$ Aggressive's state dominates OPT's state at time $t^{\prime} \geq t-i F$. We show that for all times $\tau>0$ such that Aggressive is in phase $i$ at time $t+\tau$, $c_{A}(t+\tau) \geq c_{O P T}\left(t^{\prime}+\tau\right)$, where $c_{A}(t)$ is Aggressive's cursor position at time $t$. We distinguish two cases. (1) During phase $i$, Aggressive never evicts a block from cache that is requested again in phase $i$. (2) During phase $i$, Aggressive does evict blocks that are requested again in the phase. The first case is easy to analyze. While Aggressive serves a subsequence of requests without fetching blocks, the cursor advances one request in each time step and hence OPT's cursor cannot pass Aggressive's cursor. During the service and in particular at the end of this subsequence Aggressive's holes dominate OPT's holes because the $k$ blocks in Aggressive's cache are all requested before any block not in cache and thus the holes occur at the latest possible positions. While Aggressive performs a series of fetches, Aggressive's holes always dominate OPT's holes because no further holes are introduced in the phase. Hence Aggressive's cursor cannot fall behind OPT's cursor and repeating these two arguments we obtain the desired inequality.

We next consider case (2). Let $l, 1 \leq l \leq k+\left\lfloor\frac{F}{k}\right\rfloor-1$, be the smallest index such that Aggressive, while processing the phase, evicts the block referenced by the $l$-th request in the phase. Let $t^{\prime \prime}, t^{\prime \prime} \geq t$, be the time when Aggressive initiates the prefetch in which this block is evicted. Obviously $l>k$ because when Aggressive fetches a block during the first $k$ requests it can always evict a block from cache that is not referenced during the next $k$ requests. Let $l=k+j$, for some $j \geq 1$. When Aggressive initiates the prefetch at time $t^{\prime \prime}$, there can be at most $j$ blocks missing in cache that are requested until the $l$-th request in the phase. If there were more than $j$ such blocks, then Aggressive could evict a block 
whose next request has an index larger than $k+j$. Thus the algorithm has to execute at most $j+\left\lfloor\frac{k}{F}\right\rfloor-1-j+1=$ $\left\lfloor\frac{k}{F}\right\rfloor$ fetches (including the one just initiated) to bring all blocks into cache that are still requested in the phase. We distinguish again two cases depending on whether or not these fetches are executed immediately one after the other.

Between time $t$ and $t^{\prime \prime}$ Aggressive never fetches a block that is not requested during the first $l-1$ requests of the phase. Otherwise ther would be a time where all blocks requested up to the $l$-th request are in cache and Aggressive initiates a fetch for a block that is requested after the block evicted. Thus all blocks fetched between $t$ and $t^{\prime \prime}$ were not in cache at time $t$ and using the same arguments as in case (1) we obtain that $c_{A}(t+\tau) \geq c_{O P T}\left(t^{\prime}+\tau\right)$ for all $\tau$ with $\tau \leq t^{\prime \prime}-t$. At time $t^{\prime \prime}$ the $l$-th request of the phase is at least $k$ requests away from the current request because when initiating a fetch Aggressive can always evict a block from cache that is not requested during the next $k$ references. Now suppose that the at most $\left\lfloor\frac{k}{F}\right\rfloor$ fetches after time $t^{\prime \prime}$ are executed immediately one after the other. Aggressive first fetches the $j^{\prime}, j^{\prime} \leq j$, blocks that are missing up to the $l$ th request of the phase. By the choice of $l$ all these blocks were also missing at the beginning of the phase and again Aggressive's cursor always dominates OPT's cursor during these fetches. After the service of at most $j^{\prime} F$ additional requests after time $t^{\prime \prime}$ these fetches are complete. During the next $k-j^{\prime} F \geq\left\lfloor\frac{k}{F}\right\rfloor F-j^{\prime} F=\left(\left\lfloor\frac{k}{F}\right\rfloor-j^{\prime}\right) F$ requests Aggressive can complete all the remaining fetches for blocks in the phase and hence completes these fetches before the $l$-th request. No stall time is incurred and Aggressive's cursor dominates OPT's cursor. During the rest of the phase Aggressive does not incur stall time either and again its cursor dominates OPT's cursor.

We finally consider the case that the at most $\left\lfloor\frac{k}{F}\right\rfloor$ fetches after $t^{\prime \prime}$ are not executed immediately one after the other. Let $l^{\prime}$ be the index of the last request in the phase such that at least one of the fetches still has to be executed but no fetch is performed during the service of the request. Let $s$ be the time when Aggressive reaches this request. As in the previous paragraph we can show that Aggressive's cursor dominates OPT's cursor between $t^{\prime \prime}$ and $s$ : Aggressive first fetches missing blocks that are referenced before the $l$-th request of the phase and hence were missing at the beginning of the phase. Aggressive's cursor cannot fall behind OPT's cursor. Then Aggressive fetches some blocks that are requested during the $l$-th request of the phase or later. This can be done without incurring any stall time because $l^{\prime} \leq k<l$. To see the first inequality, observe that at time $s$ the next $k$ requests are in cache because no fetch is performed; however blocks requested in the phase are still missing in cache and $\left\lfloor\frac{k}{F}\right\rfloor-1<k$. Again Aggressive's cursor cannot fall behind OPT's cursor. After time s Aggressive can fetch the at most $\left\lfloor\frac{k}{F}\right\rfloor$ missing blocks of the phase without generating any stall time. This is because $l>k$ implies $\left\lfloor\frac{k}{F}\right\rfloor-1>0$ and hence $F \leq k-1$. Aggressive's cursor dominates OPT's cursor for the rest of the phase. We conclude, as desired, $c_{A}(t+\tau) \geq c_{O P T}(t+\tau)$, for all $\tau$ such that Aggressive is still in the phase at time $t+\tau$.

The rest of the proof is identical to that of Cao et al. Let $t+t_{0}, t+t_{1}, \ldots, t+t_{r}$ be the cursor positions where Aggressive initiates fetches after time $t$ but still within the current phase. If OPT is executing a fetch at time $t+t_{q}, 0 \leq$ $q \leq r$, then let $t+t_{q}^{\prime}$ be the time when this fetch was initiated; otherwise let $t_{q}^{\prime}=t_{q}$. Cao et al. proved inductively that Aggressive's state at time $t+t_{q}$ dominates OPT's state at time $t^{\prime}+t_{q}^{\prime}$ and that Aggressive's state at time $t+t_{r}+F$ also dominates OPT's state at time $t^{\prime}+t_{r}^{\prime}+F$. The proof makes no assumptions on the phase length and only relies on the fact that $c_{A}(t+\tau) \geq c_{O P T}\left(t^{\prime}+\tau\right)$, for all $\tau$ such that Aggressive is still in phase $i$. Thus we also have that Aggressive's state at time $t+t_{r}+F$ dominates OPT's state at time $t^{\prime}+t_{r}^{\prime}+F \geq t^{\prime}+t_{r}$. We conclude that Aggressive is in phase $i+1$ at time $T=t+t_{r}+F$ and that its state dominates OPT's state at time $t^{\prime}+t_{r} \geq t-i F+t_{r} \geq T-(i+1) F$.

THEOREM 2. The approximation ratio of Aggressive is in general not smaller than $\min \left\{1+F /\left(k+\frac{k-1}{F-1}\right), 2\right\}$, for any $F>1$.

Proof. We assume $F \leq k$. For $F>k$, a lower bound of 2 was already shown by Cao et al. [5]. Consider any pair $F$ and $k$ such that $F-1$ divides $k-1$ and let $l=\frac{k-1}{F-1}$. We construct a request sequence in phases, each consisting of $k+l$ requests. In each phase we request blocks $a_{1}, \ldots, a_{k-l}$. In phase $i, i \geq$ 1 , we request $l$ new blocks $b_{1}^{i}, \ldots, b_{l}^{i}$ which have not been referenced before in the sequence. These are requested at the end of the phase. After the requests to $a_{1}$ we request the new blocks $b_{1}^{i-1}, \ldots, b_{l}^{i-1}$ from the previous phase, and these blocks will not be requested again during the rest of the sequence. Suppose that Aggressive has initially blocks $a_{1}, \ldots, a_{k-l}$ and $b_{1}^{0}, \ldots, b_{l}^{0}$ in its cache. Then the first three phases are as follows.

$$
\begin{array}{rlr}
\sigma= & a_{1}, b_{1}^{0}, \ldots, b_{l}^{0}, a_{2}, \ldots, a_{k-l}, b_{1}^{1}, \ldots, b_{l}^{1}, & / / \text { phase } 1 \\
& a_{1}, b_{1}^{1}, \ldots, b_{l}^{1}, a_{2}, \ldots, a_{k-1}, b_{1}^{2}, \ldots, b_{l}^{2}, & / / \text { phase } 2 \\
& a_{1}, b_{1}^{2}, \ldots, b_{l}^{2}, a_{2}, \ldots, a_{k-l}, b_{1}^{3}, \ldots, b_{l}^{3}, \ldots & / / \text { phase } 3
\end{array}
$$

In the first phase Aggressive starts fetching the missing blocks $b_{1}^{1}, \ldots, b_{l}^{1}$ after the service of $a_{1}$. It first evicts $a_{1}$ and then blocks $b_{1}^{0}, \ldots, b_{l-1}^{0}$ since the latter are not requested again. Aggressive needs $l \cdot F=\frac{k-1}{F-1} \cdot F=k-1+l$ time units to complete the fetches and hence has one unit of stall time before the service of $b_{l}^{1}$. Aggressive then loads the missing block $a_{1}$ by evicting $b_{l}^{0}$ and incurs $F-1$ units of stall time. At the beginning of phase 2 Aggressive has blocks $a_{1}, \ldots a_{k-l}$ and $b_{1}^{1}, \ldots, b_{l}^{1}$ in its cache. The situation is the same as at the beginning of phase 1 except that the $b_{j}^{1}$ take the role of the $b_{j}^{0}$ and the $b_{j}^{2}$ take the role of the $b_{j}^{1}, j=1, \ldots, l$. The same pattern repeats during the other phases. Thus Aggressive needs $k+l+F$ time units to serve a phase. On the other hand, an optimal strategy starts fetching the missing blocks in any phase $i$ after the service of $b_{1}^{i-1}$ and can thus evict the blocks $b_{1}^{i-1}, \ldots, b_{l}^{i-1}$ to load $b_{1}^{i}, \ldots, b_{l}^{i}$. OPT incurs two units of stall time in each phase and needs $k+l+2$ time units for any phase. The ratio of Aggressive's time to the optimal time is $1+(F-2) /\left(k+\frac{k-1}{F-1}+2\right)$ and this can be arbitrarily close to the stated bound.

In addition to the Aggressive algorithm Cao et al. [5] proposed the Conservative strategy. Conservative performs exactly the same replacements as the optimum offline paging algorithm MIN [3] while initiating a fetch at the earliest opportunity that is consistent with the choice of the block to be evicted. We now present a family of algorithms that contains Aggressive and Conservative at two ends of its spectrum. Using this family we construct an algorithm that performs 
better than Aggressive and Conservative. Let $d$ be a nonnegative integer. Intuitively the following algorithm delays a fetch for $d$ time units.

Algorithm Delay $(d)$ : Let $r_{i}$ be the next request to be served and $r_{j}, j \geq i$, the next reference where the requested block is missing in cache. If all blocks in cache are requested before $r_{j}$, serve $r_{i}$ without initiating a fetch. Otherwise let $d^{\prime}=\min \{d, j-i\}$ and let $b$ be the block whose next request is furthest in the future after request $r_{i+d^{\prime}-1}$. Initiate a fetch for $r_{j}$ at the earliest point in time after $r_{i-1}$ such the evicted block $b$ is not requested again before $r_{j}$.

Obviously, for $d=0$ we obtain the standard Aggressive strategy. For $d=n, n$ being the length of the request sequence, we obtain the Conservative algorithm. Before proving the next theorem, we mention a few implications.

Theorem 3. For any non-negative integer d, Delay(d) $\begin{array}{llll}\text { achieves and approximation } & \text { ratio } & \text { of } \\ c=\max \left\{\frac{d+F}{F}, \frac{d+2 F}{d+F}, \frac{3(d+F)}{d+2 F}\right\} & \end{array}$

Corollary 1. Setting $d_{0}=\left\lfloor\frac{1}{2}(\sqrt{3}-1) F\right\rfloor$, the approximation ratio $c_{0}$ of $\operatorname{Delay}\left(d_{0}\right)$ tends to $\sqrt{3}$.

Algorithm Combination: If $c_{0}<1+F /\left(k+\left\lfloor\frac{k}{F}\right\rfloor-1\right)$, execute Delay $\left(d_{0}\right)$, otherwise execute the standard Aggressive strategy.

Corollary 2. The approximation ratio of Combination is $\min \left\{1+F /\left(k+\left\lfloor\frac{k}{F}\right\rfloor-1\right), c_{0}\right\}$, which tends to $\min \{1+$ $\left.F /\left(k+\left\lfloor\frac{k}{F}\right\rfloor-1\right), \sqrt{3}\right\}$.

Proof of Theorem 3. In the following we call our approximation algorithm $D L$ for short, omitting the given parameter $d$. We partition the prefetching/caching schedule by $D L$ and OPT into segments $S_{D L}^{i}$ and $S_{O P T}^{i}, i \geq 1$, such that $D L$ 's state at the end of $S_{D L}^{i}$ dominates OPT's state at the end of $S_{O P T}^{i}$ and the length of $S_{D L}^{i}$ is at most $c$ times the length of $S_{O P T}^{i}$, where $c=\max \left\{\frac{d+F}{F}, \frac{d+2 F}{d+F}, \frac{3(d+F)}{d+2 F}\right\}$. This establishes the theorem. The segments $S_{D L}$ have the property that $D L$ is never in the middle of a fetch at the end of $S_{D L}^{i}$. Suppose that $S_{D L}^{1}, \ldots, S_{D L}^{i}$ and $S_{O P T}^{1}, \ldots, S_{O P T}^{i}$ have been constructed so far. Let $t$ be the time at the end of $S_{D L}^{i}$ and $t^{\prime}$ be the time at the end of $S_{O P T}^{i}$. We show how to construct the next segments $S_{D L}^{i+1}$ and $S_{O P T}^{i+1}$. If we are at the beginning of the request sequence and no segments have been constructed so far, we set $t=t^{\prime}=0$ and show how to build up the first segments.

$D L$ 's next segment starts immediately after $t$ and OPT's next segment starts immediately after $t^{\prime}$. We have to determine where the segments end and use $s$ to denote the end of $D L$ 's segment and $s^{\prime}$ to identify the end of OPT's segment. If at time $t$ all $k$ blocks in $D L$ 's cache are requested before the next missing block, the segments are easily specified. Suppose that $D L$ serves $\delta$ requests after $t$ without initiating a fetch because all blocks in cache are requested before the next missing block. Then $D L$ 's cursor at time $s=t+\delta$ dominates OPT's cursor at time $s^{\prime}=t^{\prime}+\delta$ and $D L$ 's holes at time $s$ also dominate OPT's holes at time $s^{\prime}$ because $D L$ 's holes occur at the latest possible positions. We have the desired domination and the two segments have in fact the same length.

In the following we always assume that at time $t$ there is a block in $D L$ 's cache that is referenced again only after the next block to be fetched and hence $D L$ can initiate a fetch. Assume that $D L$ needs $D_{1}, D_{1} \leq d+F$, time units after $t$ to complete the next fetch. If OPT does not initiate a fetch during the next $D_{1}$ time units after $t^{\prime}$, then we are done. $D L$ 's cursor at time $s=t+D_{1}$ dominates OPT's cursor at time $s^{\prime}=t^{\prime}+D_{1}$. This is obvious if $D L$ does not incur stall time to complete the fetch. If $D L$ does incur stall time, then $D L$ fetches the block referenced right after $t+D_{1}$. OPT's cursor cannot pass $D L$ 's cursor because $D L$ 's holes at time $t$ dominate OPT's holes at time $t^{\prime}$. Since OPT's holes do not change between $t^{\prime}$ and $s^{\prime} D L$ 's holes at time $s$ also dominate OPT's holes at time $s^{\prime}$. Again we have the desired domination and $D L$ 's and OPT's segments have the same length.

We therefore assume in the following that OPT initiates a fetch during the next $D_{1}$ time units after $t^{\prime}$. Suppose that $D L$ serves exactly $d_{1}$ requests after $t$ and that OPT serves $d_{1}^{\prime}$ after $t^{\prime}$ before initiating the next fetch. If $d_{1}^{\prime} \leq d_{1}$, the analysis is simple. $D L$ 's state at time $t+d_{1}$ dominates OPT's state at time $t^{\prime}+d_{1}^{\prime}$ and by the Lemma $1 D L$ 's state at time $s=t+d_{1}+F=t+D_{1}$ dominates OPT's state at time $s^{\prime}=t^{\prime}+d_{1}^{\prime}+F$. The ratio of $D L$ 's segment length to OPT's segment length is at most $D_{1} /\left(d_{1}^{\prime}+F\right) \leq(d+F) / F$. If $d_{1}^{\prime}>d_{1}$ but $d_{1}^{\prime} \leq d$, then let $r_{i}$ be the next request to be served by $D L$ and $r_{j}$ be the location of the next hole at time $t$. Set $\bar{d}=\min \left\{j-i, d_{1}^{\prime}\right\}$. Imagine we would modify $D L$ as follows. After time $t D L$ serves $\bar{d}$ requests before initiating a fetch for $r_{j}$. During this fetch it evicts the block whose next reference is furthest in the future. Since $D L$ 's state at time $t$ dominates OPT's state at time $t^{\prime}$, the modified algorithm's state at time $t+\bar{d}$ dominates OPT's state at time $t+d_{1}^{\prime}$. By Lemma 1 the modified algorithm's state at time $t+\bar{d}+F$ dominates OPT's state at time $t^{\prime}+d_{1}^{\prime}+F$. By definition the original $D L$ algorithm may delay a fetch for $d$ requests and hence the block evicted during the first fetch after $t$ is equal to the block evicted by the modified algorithm during the first fetch after $t$. We obtain that $D L$ 's holes at time $s=t+D_{1}$ dominate OPT's holes at time $t^{\prime}+d_{1}^{\prime}+F$, which are equal to OPT's holes at time $s^{\prime}=\min \left\{t^{\prime}+D_{1}, t^{\prime}+d_{1}^{\prime}+\right.$ $F\}$. Also, $D L$ 's cursor at time $s$ dominates OPT's cursor at time $s^{\prime}$ because if $D L$ incurs stall time to complete the fetch then OPT's cursor cannot pass because its holes were dominated by $D L$ 's holes. In summary we have domination and the ratio of the segment length is upper bounded by $D_{1} / F \leq(d+F) / F$.

In the remainder of this proof we assume $d_{1}^{\prime}>d$. If at time $t+D_{1}$ the $k$ blocks in $D L$ 's cache are all referenced before the next missing block, then the segments are easily determined. OPT needs $D_{1}^{\prime}=d_{1}^{\prime}+F$ time units to complete the first fetch after $t^{\prime}$. If $D L$ does not incur stall time to complete the first fetch, then its cursor at time $s=t+D_{1}$ dominates OPT's cursor at time $t^{\prime}+D_{1}$. If $D L$ does incur stall time, then OPT's cursor cannot pass $D L$ 's cursor during the first fetch because $D L$ 's holes at time $t$ dominate OPT's holes at time $t^{\prime}$. In this case $D L$ 's cursor at time $s$ dominates OPT's cursor at time $t^{\prime}+D_{1}$. Thus $D L$ 's cursor at time $s$ dominates OPT's cursor at time $s^{\prime}=\min \left\{t^{\prime}+D_{1}, t^{\prime}+D_{1}^{\prime}\right\}$ and $D L$ 's holes at time $s$ dominate OPT's holes at time $s^{\prime}$ because $D L$ 's holes occur at the latest possible positions. The ratio of $D L$ 's segment length to OPT's segment length is at most $D_{1} / F \leq(d+F) / F$ because $F \leq D_{1} \leq d+F$ and $D_{1}^{\prime} \geq F$

It remains to analyze the case that $d_{1}^{\prime}>d$ and at time 
$t+D_{1}$ there is a block in $D L$ 's cache that is referenced after the next missing block. Let $D_{2}=d_{2}+F$ be the number of time units after $t+D_{1} D L$ needs to complete the next fetch. We have $d_{2} \leq d$ by the definition of $D L$. We distinguish two cases. (1) $d_{1}+d_{2} \leq d_{1}^{\prime}$ and (2) $d_{1}+d_{2}=d_{1}^{\prime}+\delta$ for some positive integer $\delta$. We first consider case (1). We have that $D L$ 's state at time $t+D_{1}$ dominates OPT's state at time $t+d_{1}^{\prime}$. The reason is that $D L$ 's cursor at time $t+D_{1}$ dominates OPT's cursor at time $t+d_{1}^{\prime}$ because OPT initiates the first fetch after $t^{\prime}$ within the next $D_{1}$ time units and $D L$ 's holes at time $t$ dominate OPT's holes at time $t^{\prime}$, i.e. OPT's cursor cannot pass $D L$ 's cursor during the first fetch. Since OPT's holes do not change between $t^{\prime}$ and $t^{\prime}+d_{1}^{\prime}, D L$ 's holes at time $t+D_{1}$ also dominate OPT's holes at time $t+d_{1}^{\prime}$. Since $D L$ 's state at time $t+D_{1}$ dominates OPT's state at time $t+d_{1}^{\prime}, D L$ 's state at time $t+D_{1}+d_{2}$ also dominates OPT's state at time $t+d_{1}^{\prime}$ and by Lemma $1 D L$ 's state at time $s=t+D_{1}+d_{2}+F=t+D_{1}+D_{2}$ dominates OPT's state at time $s^{\prime}=t^{\prime}+d_{1}^{\prime}+F=t^{\prime}+D_{1}^{\prime}$. The ratio of the segment lengths is $\left(D_{1}+D_{2}\right) / D_{1}^{\prime} \leq\left(d_{1}^{\prime}+2 F\right) /\left(d_{1}^{\prime}+F\right) \leq$ $(d+2 F) /(d+F)$.

We next study case (2). First observe that $D L$ 's cursor at time $t+D_{1}+d_{2}$ dominates OPT's cursor at time $t^{\prime}+D_{1}+d_{2}$. This is obvious if $D L$ does not incur stall time to complete the first fetch. If $D L$ does incur stall time, then $D L$ 's cursor at time $t+D_{1}$ must dominate OPT's cursor at time $t^{\prime}+D_{1}^{\prime} \geq$ $t^{\prime}+D_{1}$ because $D L$ 's holes at time $t$ dominate OPT's holes at time $t^{\prime}$ and OPT cannot finish the first fetch later in the sequence than $D L$. Since $D L$ 's cursor advances one step in each of the following $d_{2}$ time units after $t+D_{1}$ we have the stated domination for the cursors. If OPT does not initiate a second fetch before $t^{\prime}+D_{1}+d_{2}$, then we are done. As in case (1) we have that $D L$ 's state at time $s=t+D_{1}+D_{2}$ dominates OPT's state at time $t^{\prime}+D_{1}^{\prime}=t^{\prime}+D_{1}+d_{2}-\delta$. This implies that $D L$ 's state at time $s$ dominates OPT's state at time $s^{\prime}=t^{\prime}+D_{1}+d_{2}$ because $D L$ 's cursor at time $s>t+D_{1}+d_{2}$ dominates OPT's cursor at time $t^{\prime}+D_{1}+d_{2}$ as shown above and OPT's holes do not change between $t^{\prime}+D_{1}+d_{2}-\delta$ and $s^{\prime}$. The ratio of the segment lengths is $\left(D_{1}+D_{2}\right) /\left(D_{1}+d_{2}\right)=$ $\left(d_{1}^{\prime}+\delta+2 F\right) /\left(d_{1}^{\prime}+\delta+F\right) \leq(d+2 F) /(d+F)$. If OPT does initiate a second fetch before $t^{\prime}+D_{1}+d_{2}$ but at time $t+D_{1}+D_{2}$ all $k$ block in $D L$ 's cache are all requested before the next missing block, then $D L$ 's state at time $s=$ $t+D_{1}+D_{2}$ dominates OPT's state at time $s^{\prime}=t^{\prime}+D_{1}+d_{2}$ because $D L$ 's holes occur at the latest possible positions. The ratio of $D L$ 's segment length to OPT's segment length is upper bounded by $\left(D_{1}+D_{2}\right) /\left(D_{1}+d_{2}\right) \leq(d+2 F) /(d+F)$.

We finally have to consider the case that OPT initiates a second fetch before $t^{\prime}+D_{1}+d_{2}$ but at time $t+D_{1}+D_{2}$ there is a block $D L$ 's cache that is requested after the next missing block. $D L$ needs $D_{3}=d_{3}+F$ time units with $d_{3} \leq d$ to complete the next fetch. Suppose that OPT initiates the second fetch at time $t^{\prime}+D_{1}^{\prime}+\delta^{\prime}$, with $\delta^{\prime} \leq \delta$. As above we have that $D L$ 's state at time $t+D_{1}+D_{2}$ dominates OPT's state at time $t^{\prime}+D_{1}^{\prime}$. This implies that $D L$ 's state at time $t+D_{1}+D_{2}$ dominates OPT's state at time $t^{\prime}+D_{1}^{\prime}+\delta^{\prime}$ because $D L$ 's cursor at time $t+D_{1}+D_{2}$ dominates OPT's cursor at time $t^{\prime}+D_{1}^{\prime}+\delta \geq t^{\prime}+D_{1}^{\prime}+\delta^{\prime}$ and OPT's holes do not change between $t^{\prime}+D_{1}^{\prime}$ and $t^{\prime}+D_{1}^{\prime}+\delta^{\prime}$. It follows that $D L$ 's state at time $t+D_{1}+D_{2}+d_{3}$ dominates OPT's state at time $t^{\prime}+D_{1}^{\prime}+\delta^{\prime}$ and by Lemma $1 D L$ 's state at time $s=t+D_{1}+D_{2}+d_{3}+F$ dominates OPT's state at time $s^{\prime}=t^{\prime}+D_{1}^{\prime}+\delta^{\prime}+F$. $D L$ 's segment length is at most
$3(d+F)$ while OPT's segment length is at least $(d+2 F)$.

\section{MINIMIZING STALL TIME IN PARAL- LEL DISK SYSTEMS}

In this section we present a polynomial time algorithm for systems with $D$ parallel disks that, given a request sequence $\sigma$, computes a prefetching/caching schedule whose stall time is at most that of an optimal solution. The schedule uses not more than $3(D-1)$ extra memory locations in cache.

The basic idea is to use the linear programming approach of [1] but to model the objective function, which measures the stall time of a schedule, in a different way. For this purpose we consider synchronized schedules that are defined as follows. Consider a prefetching/caching schedule for $\sigma$. A fetch operation executed from time $t_{1}$ to time $t_{1}^{\prime}$ intersects a fetch operation performed from $t_{2}$ to $t_{2}^{\prime}$ if there is a $t$ with $t_{1} \leq t \leq t_{1}^{\prime}$ and $t_{2} \leq t \leq t_{2}^{\prime}$ but $t_{1} \neq t_{2}$ (and hence $\left.t_{1}^{\prime} \neq t_{2}^{\prime}\right)$. Clearly, fetch operations executed on the same disk cannot intersect. A prefetching/caching schedule is synchronized if no two fetch operations intersect. Intuitively, in a synchronized schedule fetch operations on different disks are executed completely in parallel, starting and ending at exactly the same time. For a given $\sigma$, let $s_{O P T}(\sigma)$ be the stall time of an optimal schedule for $\sigma$. We show that there exist synchronized schedules that achieve a minimum stall time provided that they may use up to $D-1$ extra cache locations.

Lemma 2. For any $\sigma$, there exists a synchronized schedule that achieves a stall time of at most $s_{O P T}(\sigma)$ and uses not more than $D-1$ extra memory locations in cache.

Proof. Let $S$ be an optimal prefetching/caching schedule using $k$ cache locations. We show how to modify $S$ so that the resulting schedule is synchronized and the stall time does not increase. Suppose that (a) up to time $t$ schedule $S$ is synchronized and uses at most $D-1$ extra cache locations and (b) from time $t$ on the schedule is not synchronized but uses no extra cache locations. Moreover assume that at time $t$ a fetch operation is initiated that intersects fetches on other disks. (Initially, $t$ is the first point in time at which a fetch operation intersecting other fetches starts.) Let $t^{\prime}$ be the time when the fetch ends. Suppose that the fetch from $t$ to $t^{\prime}$ intersects $d, 1 \leq d \leq D-1$, fetches on other disks. Let $t_{1}, \ldots, t_{d}$ be the times when these fetches start. Furthermore, let $a_{1}, \ldots, a_{d}$ be the blocks fetched and $b_{1}, \ldots, b_{d}$ be the blocks evicted during these fetch operations. The schedule is now modified as follows. We delete the fetch operations initiated at times $t_{1}, \ldots, t_{d}$ and instead fetch $a_{1}, \ldots, a_{d}$ into the $D-1$ available extra cache locations starting at time $t$. At time $t^{\prime}$, when these fetches end, we evict $b_{1}, \ldots, b_{d}$ from cache so that the $D-1$ extra cache locations are again available. The stall time does not increase during this modification because a possible stall time incurred at the end of the fetch at time $t^{\prime}$ was already needed for the original fetch from $t$ to $t^{\prime}$. At the end of the fetch blocks $b_{1}, \ldots, b_{d}$ are available for eviction because $b_{i}$ was available at time $t_{i} \leq t^{\prime}, 1 \leq i \leq d$. From time $t^{\prime}$ on the schedule uses only $k$ cache locations. Repeating this step for times $t, t>t^{\prime}$, at which intersecting fetches are initiated, we obtain a synchronized schedule with stall time at most $s_{O P T}(\sigma)$. 
We now describe a 0-1 linear program for computing an optimal synchronized prefetching/caching schedule that uses $k+D-1$ cache locations. Let $n$ be the number of requests in the given sequence $\sigma$. The linear program has to determine the intervals in which the synchronized fetches are performed. As in [1] we consider intervals $I=(i, j)$ of length at most $F$ in the request sequence, $i=0, \ldots, n-1$ and $j=1, \ldots, n$. The length of an interval is $|I|=j-i-1$. Such an interval represents a fetch that starts after request $r_{i}$ and ends before $r_{j}$. Since a fetch takes $F$ time units, $F-|I|$ units of stall time are incurred at the end of $I$. For each such interval we introduce a variable $x(I)$ that is 1 if (synchronized) fetches are performed in interval $I$ and 0 otherwise. The stall time of a synchronized schedule is easy to compute; it is just the sum of the stall times incurred at the end of fetch intervals. Thus we wish to minimize $\sum_{I} x(I)(F-|I|)$.

The rest of the linear program is similar to that given in [1], except that several constraints simplify. We say that in interval $(i, j)$ is properly contained in an interval $\left(i^{\prime}, j^{\prime}\right)$, i.e. $(i, j) \subseteq\left(i^{\prime}, j^{\prime}\right)$, if $i \geq i^{\prime}$ and $j \leq j^{\prime}$. We have to ensure that at any time only one set of synchronized fetches is performed. Therefore, for any $i$ with $1 \leq i \leq n-1$ we add the constraint $\sum_{(i-1, i+1) \subseteq I} x(I) \leq 1$.

The linear program also has to determine the blocks to be fetched and evicted in each interval. We assume without loss of generality that the cache initially contains a set $S_{\text {init }}$ of $k+D-1$ blocks from disk 1 which are never requested in $\sigma$. Let $S_{d}$ be the set of blocks in $\sigma$ that are stored on disk $d$, $1 \leq d \leq D$, and let $S=S_{1} \cup \ldots \cup S_{D} \cup S_{\text {init }}$. For any interval $I$ and any block $a \in S$ we introduce a variable $f_{I, a}$ that is 1 if $a$ is fetched in interval $I$ and 0 otherwise. Furthermore, for any $I$ and any block $a \in S$ there is a variable $e_{I, a}$ that is 1 if $a$ is evicted in $I$ and 0 otherwise. We have to ensure that, for any interval $I$ and any disk $d, 1 \leq d \leq D$, only one block from disk $d$ is fetched. Of course such a fetch can only be performed if $x(I)=1$. Thus we add

$$
\forall I, d \sum_{a \in S_{d}} f_{I, a} \leq x(I) .
$$

We also have to make sure that in each interval the number of blocks fetched is equal to the number of blocks evicted, i.e. we have

$$
\forall I \sum_{a \in S} f_{I, a}=\sum_{a \in S} e_{I, a}
$$

When a request is served, the requested block must be in cache. For any $a \in S_{1} \cup \ldots \cup S_{D}$ let $i_{1}<i_{2}<\ldots<i_{l}$ be the indices of the requests to $a$. We add the constraints $\sum_{I \subset\left(0, i_{1}\right)} f_{I, a}=1$ and $\sum_{I \subseteq\left(0, i_{1}\right)} e_{I, a}=0$, which guarantee that $a$ is in cache at the time of its first request. We additionally impose, for $j=1, \ldots, l-1$,

$$
\sum_{I \subseteq\left(i_{j}, i_{j+1}\right)} f_{I, a}=\sum_{I \subseteq\left(i_{j}, i_{j+1}\right)} e_{I, a} \leq 1,
$$

which implies that if $a$ is in cache at the time of its $j$ th reference then it is also in cache at the time of its $(j+1)$ st reference. Finally we have $\sum_{I \subset\left(i_{l}, n\right)} e_{I, a} \leq 1$. Of course, a block may not be fetched or evicted when it is referenced. Thus we have, for $j=1, \ldots, l$,

$$
\sum_{I:\left(i_{j}-1, i_{j}+1\right)} f_{I, a}=\sum_{I:\left(i_{j}-1, i_{j}+1\right)} e_{I, a}=0 .
$$

With respect to the blocks $a \in S_{\text {init }}$ we only require $\sum_{I \subseteq(0, n)} e_{I, a} \leq 1$.

We have $n \min \{F+1, n\}$ variables $x(I)$ and $O\left(n^{2} \min \{F+\right.$ $1, n\})$ variables $f_{I, a}$ and $e_{I, a}$. Note that we can assume $k \leq n$ since otherwise we could simply load the requested blocks into cache and then serve all requests. Also, we can assume $D \leq n$ because otherwise we just ignore the disks that do not contain a block requested in $\sigma$. Relaxing the 0 -1 variables to $0 \leq x(I), f_{I, a}, e_{I, a} \leq 1$, we can compute in polynomial time a solution whose value is bounded by $s_{O P T}(\sigma)$. The idea of the following analysis is to show that a fractional solution to the relaxed linear program is a convex combination of polynomially many integral solutions. We can then select one of these integral solutions and achieve a minimum stall time.

Let $\mathcal{I}=\{I \mid x(I)>0\}$. As is [1] we can modify the fractional solution such that for any two intervals $I=(i, j)$ and $I^{\prime}=\left(i^{\prime}, j^{\prime}\right)$ in $\mathcal{I}$ with $I \subseteq I^{\prime}$ we have $i=i^{\prime}$ or $j=j^{\prime}$, i.e. intervals share a common endpoint if one is properly contained in the other. Based on this relation we can define a linear order $<$ on $\mathcal{I}$. The intervals are ordered by increasing startpoints and, if intervals have the same startpoint, they are ordered by increasing endpoints.

In order to be able to apply techniques from [1] it is crucial that in each interval $I \in \mathcal{I}$ all $D$ disks fetch an amount of exactly $x(I)$. Clearly, there is at least one disk $d$ with $\sum_{a \in S_{d}} f_{I, a}=x(I)$ since otherwise we could decrease $x(I)$. To establish this property for all $I$ and $d$, we schedule dummy fetches on the idle disks in $I$. Since these fetches must not change the configuration of the $k+D-1$ cache locations, we introduce $D-1$ additional cache positions that initially contain $D-1$ blocks $b_{1}^{0}, \ldots, b_{D-1}^{0}$ from disk 1 which are never requested in $\sigma$. We then consider the intervals in $\mathcal{I}$ in the order of $<$. Let $I$ be the $j$ th interval considered. For any of the at most $D-1$ disks $d$ with $\delta_{d}=x(I)-\sum_{a \in S_{d}} f_{I, a}>0$ we fetch a new block $b_{d}^{j}$ from disk $d$ to an extent of $\delta_{d}$ and evict an amount of $\delta_{d}$ of the blocks $b_{1}^{l}, \ldots, b_{D-1}^{l}$ with the smallest index $l$ that reside in the extra $D-1$ cache locations. Blocks $b_{d}^{j}, 1 \leq d \leq D$ and $j>0$ are never requested in $\sigma$. The dummy blocks keep disks busy that are originally idle. It is sufficient to use at $D-1$ cache locations because, as mentioned before, in each interval there is at least one disk that fetches to an extent of $x(I)$.

We modify the optimal fractional solution even further. More specifically, it is an easy exercise to show that there is an optimal fractional solution that satisfies the following properties on the fetches and evictions. Consider the intervals in the order $<$ and let $C$ denote the cache configuration after we have performed fetches and evictions corresponding to the first $j$ intervals in the order. Let $I$ be the $(j+1)$ st interval.

- For any $d, 1 \leq d \leq D$, we fetch the block from disk $d$ that is not completely in $C$ and whose next reference is earliest.

- If we evict a block from disk $d$ in $I$, then it is the block from disk $d$ which is partially or completely in $C$ and whose next reference is furthest in the future.

Based on these properties it is possible to view the prefetching/caching schedule as a process over time. For any $I \in \mathcal{I}$, define $\operatorname{dist}(I)=\sum_{I^{\prime}<I} x\left(I^{\prime}\right)$, i.e. $\operatorname{dist}(I)$ is the sum of the $x\left(I^{\prime}\right)$ where $I^{\prime}$ precedes $I$ in the order $<$. The time interval associated with $I$ is $[\operatorname{dist}(I), \operatorname{dist}(I)+x(I)]$. Hence 
there is a unique interval $I$ associated with each time. For any interval $I \in \mathcal{I}$ and any disk $d, 1 \leq d \leq D$, we sort the blocks fetched from disk $d$ in $I$ by increasing order of their next reference. Let $a_{1}, \ldots, a_{l}$ be the blocks in this order. Block $a_{i}$ is fetched for $f_{I, a_{i}}$ time units starting at time $\operatorname{dist}(I)+\sum_{j=1}^{i-1} f_{i, a_{j}}$. Hence at each time instant we fetch a unique block from each disk.

As in [1], for any $t$ in the range $[0,1)$, we construct an integral feasible solution that uses $D-1$ cache locations in addition to the $k+2(D-1)$ locations we already use. Let $\mathcal{I}_{t}$ be the set of intervals $I$ in $\mathcal{I}$ associated with time instances $t_{i}=t+i$, for all $i \geq 0$. Each interval $I$ in $\mathcal{I}_{t}$ is part of the solution for $t$. If $I \in \mathcal{I}_{t}$ is the interval associated with time $t_{i}$, then for any disk $d$ we fetch the block that is loaded from disk $d$ at time $t_{i}$. The algorithm for assigning evictions is slightly different from the one described in [1]. We maintain a set $Q_{t}$ that is initially empty and consider the intervals in $\mathcal{I}$ in the order $<$. Let $I$ be the current interval and $a_{1}, \ldots, a_{l}$ be the blocks evicted in $I$. If $a_{j}, 1 \leq j \leq l$, is fetched back at time $t_{i}$, for some $i \geq 0$, before its next reference, then add $a_{j}$ to $Q_{t}$. If $I \in \mathcal{I}$ and $Q_{t}$ currently contains at least $D$ blocks, then remove $D$ arbitrary blocks from $Q_{t}$ and evict them during $I$. If $Q_{t}$ currently contains less than $D$ blocks, then remove only these available blocks and evict them in $I$.

Lemma 3. For any $t \in[0,1)$, solution $\mathcal{I}_{t}$ is an integral feasible solution that uses a total of at most $k+3(D-1)$ cache locations.

Proof. The intervals in $\mathcal{I}_{t}$ are disjoint. Moreover, by the definition of our algorithm for scheduling evictions, each block that is assigned to $Q_{t}$ and hence evicted in an interval of $\mathcal{I}_{t}$ is also fetched back before its next reference in an interval of $\mathcal{I}_{t}$. Hence $\mathcal{I}_{t}$ is a feasible solution. The optimal fractional solution used to construct $\mathcal{I}_{t}$ uses $2(D-1)$ extra memory locations in cache. We will show that at most $D-1$ intervals in $\mathcal{I}_{t}$ do not have an eviction assigned. If we load the blocks fetched in those intervals into $D-1$ extra memory locations, then $\mathcal{I}_{t}$ is a feasible solution that uses at most $3(D-1)$ extra cache locations.

Consider our algorithm for scheduling evictions and suppose that we just finished processing interval $I \in \mathcal{I}$. For any disk $d$ let $s_{d}$ the last point in time such that disk $d$ fetches a block that has been evicted in intervals $I^{\prime} \leq I$ but not yet been fetched back at time instances corresponding to $I^{\prime} \leq I$. Suppose that there exists a time before $s_{d}$ such that disk $d$ fetches a block that has not yet been evicted in intervals $I^{\prime} \leq I$. Let $s_{d}^{\prime}$ be the earliest point in time with the property and let $a_{d}$ be the block fetched at this point in time. Let $b_{d}$ be any block that has been evicted in intervals $I^{\prime} \leq I$ and is fetched back after $s_{d}^{\prime}$. Since $b_{d}$ is fetched after $a_{d}$ the next reference to $b_{d}$ must be after the next reference to $a_{d}$. The last eviction of $b_{d}$ in intervals $I^{\prime} \leq I$ must be an eviction where $b_{d}$ is discarded to an extent of 1 . If $b_{d}$ were discarded only partially, then our optimal fractional solution would have evicted the rest of $b_{d}$ in the operations where $a_{d}$ is evicted because $b_{d}$ 's next reference is later. Thus when $b_{d}$ is fetched back after $s_{d}^{\prime}$ it is fetched back to an extent of 1 and this fetch is performed continuously without interruption. This implies that our algorithm added $b_{d}$ to $Q_{t}$. Let $E_{d}$ be the total amount of evictions of blocks from disk $d$ up to the current interval $I$, i.e. $E_{d}=\sum_{a \in S_{d}} \sum_{I^{\prime} \leq I} e_{I^{\prime}, a}$. Part of this amount is fetched back continuously until time $s_{d}^{\prime}$. Blocks fetched back later are, as mentioned before, fetched to an extent of 1 and added to $Q_{t}$. Hence, when the algorithm finishes processing $I,\left\lfloor E_{d}-t\right\rfloor+1$ blocks from disk $d$ have been assigned to $Q_{t}$ and summing over all disks a total of $\sum_{d=1}^{D}\left(\left\lfloor E_{d}-t\right\rfloor+1\right)$ blocks have been assigned to $Q_{t}$. Let $X(I)=\sum_{I^{\prime} \leq I} x\left(I^{\prime}\right)$. When the algorithm finishes processing $I$, it has tried to assign $D(\lfloor X(I)-t\rfloor+1)$ evictions because $\mathcal{I}_{t}$ contains $\lfloor X(I)-t\rfloor+1$ intervals $I^{\prime}$ with $I^{\prime} \leq I$ in each of which we schedule $D$ fetches and evictions. Moreover, $X(I)=\sum_{d=1}^{D} E_{d}$ because in our fractional solution in each interval $I^{\prime}$ the amount of fetches and evictions is exactly $x\left(I^{\prime}\right)$. Hence

$$
\begin{aligned}
& D(\lfloor X(I)-t\rfloor+1)-\sum_{d=1}^{D}\left(\left\lfloor E_{d}-t\right\rfloor+1\right) \\
\leq & D(X(I)-t+1)-\sum_{d=1}^{D}\left(E_{d}-t+1\right)+D-1 \\
= & D-1 .
\end{aligned}
$$

We conclude that at most $D-1$ fetch operations on the various disks to not get an eviction assigned.

When constructing the solutions $\mathcal{I}_{t}$ as $t$ varies from 0 to 1 , we obtain a given solution not for just one value of $t$ but for a range of values. Let $0=x_{1}<x_{2}<\ldots<x_{l}=1$ be the set of values such that for all $t$ in the range $\left[x_{i}, x_{i+1}\right)$ we obtain the same solution $\mathcal{I}_{t}, 1 \leq i<l$. Hence $\mathcal{I}_{x_{1}}, \ldots, \mathcal{I}_{x_{l-1}}$ are the different solutions we obtain. Since each $\mathcal{I}_{x_{j}}, 1 \leq$ $j \leq l-1$, is a synchronized schedule its stall time $s\left(\mathcal{I}_{x_{j}}\right)$ is equal to the sum of the stall times incurred by the intervals in $\mathcal{I}_{x_{j}}$. Giving $\mathcal{I}_{x_{j}}$ a weight of $x_{j+1}-x_{j}$, we obtain that $\sum_{j=1}^{l-1}\left(x_{j+1}-x_{j}\right) s\left(\mathcal{I}_{x_{j}}\right)$ is equal to the value of the optimal fractional solution. It follows that one of the $\mathcal{I}_{x_{j}}$ achieves a stall time that is bounded by the value of the optimal fractional solution and hence bounded by the minimum stall time for $\sigma$. Finding such an $\mathcal{I}_{x_{j}}$ is easy and in fact we do not even have to compute explicitely all the $\mathcal{I}_{x_{1}}, \ldots, \mathcal{I}_{x_{l-1}}$. All we have to do is to compute a $t_{0}$ such that the total stall time of intervals in $\mathcal{I}_{t_{0}}$ is minimum among all $\mathcal{I}_{t}$. For varying $t$, the intervals in $\mathcal{I}_{t}$ only change if an interval $I \in \mathcal{I}$ starts at some time $t_{i}$. Thus we only have to check $|\mathcal{I}|=$ $O(n \min \{F+1, n\})$ values of $t$. Once we have determined an optimal $t_{0}$, we apply our algorithm to schedule the evictions. This establishes our main result.

THEOREM 4. There exists a polynomial time algorithm for integrated prefetching and caching on $D$ parallel disks that, given a request sequence $\sigma$, computes a schedule whose stall time is at most that of an optimal solution for $\sigma$. The schedule uses at most $3(D-1)$ extra memory locations in cache.

\section{CONCLUSIONS}

In this paper we presented improved prefetching/caching algorithms for single and parallel disk systems. In the single disk setting an interesting problem is to develop fast algorithms that achieve an even smaller approximation ratio with respect to the elapsed time performance measure. A challenging open problem is to determine the complexity of the parallel disk case: Is it NP-hard to construct optimal schedules or does there exist a polynomial time algorithm? 


\section{REFERENCES}

[1] S. Albers, N. Garg and S. Leonardi. Minimizing tall time in single and parallel disk systems. Journal of the ACM, 47:969-986, 2000.

[2] S. Albers and C. Witt. Minimizing stall time in single and parallel disk systems using multicommodity network flows. Proc. 4th International Workshop on Approximation Algorithms for Combinatorial Optimization Problems APPROX), Springer LNCS 2129, 12-23, 2001.

[3] L.A. Belady. A study of replacement algorithms for virtual storage computers. IBM Systems Journal, 5:78$101,1966$.

[4] A. Borodin, S. Irani, P. Raghavan and B. Schieber. Competitive paging with locality of reference. Journal on Computer and System Sciences, 50:244-258, 1995.

[5] P. Cao, E.W. Felten, A.R. Karlin and K. Li. A study of integrated prefetching and caching strategies. Proc. ACM International Conference on Measurement and Modeling of Computer Systems (SIGMETRICS), 188196, 1995

6] P. Cao, E.W. Felten, A.R. Karlin and K. Li. Implementation and performance of integrated applicationcontrolled caching, prefetching and disk scheduling. ACM Transaction on Computer Systems (TOCS), 14:311-343, 1996.

[7] A. Fiat and M. Mendel. Truly online paging with locality of reference. Proc. 38th IEEE Symposium on Foundations of Computer Science, 1997.

[8] D.R. Fuchs and D.E. Knuth. Optimal prepaging and font caching. ACM Transactions on Programming Languages and Systems, 7:62-79, 1985.

[9] A. Gaysinsky, A. Itai, and H. Shachnai. Strongly competitive algorithms for caching with pipelined prefetching. Proc. 9th Annual European Symposium on Algorithms (ESA01), Springer LNCS 2161, 49-61, 2001.

[10] D.A. Hutchinson, P. Sanders, and J.S. Vitter. Duality between prefetching and queued writing with parallel disks. Proc. 9th Annual European Symposium on Algorithms (ESA01), Springer LNCS 2161, 62-73, 2001.

[11] M. Kallahalla and P.J. Varman. Optimal prefetching and caching for parallel I/O systems. Proc. 13th ACM Symposium on Parallel Algorithms and Architectures, 2001.

[12] T. Kimbrel and A.R. Karlin. Near-optimal parallel prefetching and caching. SIAM Journal on Computing, 29:1051 - 1082, 2000. Preliminary version in FOCS96.

[13] T. Kimbrel, P. Cao, E.W. Felten, A.R. Karlin and K. Li. Integrated parallel prefetching and caching. Proc. ACM International Conference on Measurement and Modeling of Computer Systems (SIGMETRICS), 1996.

[14] T. Kimbrel, A. Tomkins, R.H. Patterson, B. Bershad, P. Cao, E.W. Felten, G.A. Gibson, A.R. Karlin and K. Li. A trace-driven comparison of algorithms for parallel prefetching and caching. Proc. of the $A C M$ SIGOPS/USENIX Association Symposium on Operating System Design and Implementation, 1996.

[15] P. Krishnan and J.S. Vitter. Optimal prediction for prefetching in the worst case. SIAM Journal on Computing, 27:1617-1636, 1998.

[16] M. Palmer and S.B. Zdonik. Fido: A cache that learns to fetch. Proc. 17th International Conference on Very Large Data Bases, 255-264, 1991.
[17] R.H. Patterson, G.A. Gibson, E. Ginting, D. Stodolsky and J. Zelenka. Informed prefetching and caching. Proc. 15th Symposium on Operating Systems Principles, 7995, 1995.

[18] D.D. Sleator and R.E. Tarjan. Amortized efficiency of list update and paging rules. Communication of the $A C M, 28: 202-208,1985$.

[19] J. Vitter and P. Krishnan. Optimal prefetching via data compression. Journal of the ACM, 43:771-793, 1996. 